\title{
Contribution à l'identification des sols effondrables
}

A contribution to the identification of collapsible soils

K. ABBECHE

Département de génie civil Université de Batna

Rue Boukhlouf

05000 Batna Algérie

L. MOKRANI

Département de génie civil

Université de Sétif

019000 Sétif Algérie

A. BOUMEKIK

Département de génie civil

Université de Constantine

025000 Constantine Algérie

\section{Résumé}

L'étude de l'effondrement des sols sous l'effet de l'inondation est un problème majeur en mécanique des sols. La majorité des travaux effectués sur les paramètres qui gouvernent l'effondrement des sols ont été consacrés à l'influence de la densité sèche initiale, de la teneur en eau, du degré de saturation et de la charge appliquée. Peu de travaux ont concerné l'étude de l'influence de la compacité, de l'indice de consistance et de la fraction argileuse $(\%<2 \mu \mathrm{m})$. Cette étude expérimentale conduite sur des sols reconstitués a pour but d'étudier l'influence de ces paramètres sur l'effondrement et de proposer une méthode d'identification de ce type de sols.

Mots clés: sols effondrables, indice de densité, indice de consistance, fraction argileuse.

\section{Introduction}

Des désordres importants causés par des effondrements (ou affaissements) de sols sous l'effet d'infiltrations d'eau ont été observés dans plusieurs régions du monde, notamment en zones arides ou semi-arides. Récemment, les observations faites dans les régions de Biskra et d'Hassi Messaoud (Algérie) en constituent une bonne illustration.

A Biskra, plusieurs bâtiments d'habitation ont subi des dégradations importantes causées par un effondrement du sol essentiellement dû aux infiltrations des eaux (CTC Biskra, 2002). Lors de l'étude géotechnique pour la réalisation d'une station de compression du gaz naturel à Hassi Messaoud, il a été observé que les sols de fondation présentaient une nature effondrable. Cette étude a montré que le site était composé de deux couches.

La première couche, de 3 m d'épaisseur, en limon argileux, possède les caractéristiques suivantes: teneur en eau $\mathrm{w}_{0}=5,6 \%$, degré de saturation

\section{Abstract}

The study of the collapse of soils under wetting is a major problem in soil mechanics. Most of the research works conducted on the parameters that govern soil collapse have been concentrated on the effects of the initial dry density, water content and applied load. Few works have been devoted to the influence of compacity, of the consistency index and of the clay fraction $(\%<2 \mu \mathrm{m})$ on the soil collapse susceptibility. This experimental study, conducted on reconstituted soils, considers the influence of these parameters on collapse soils and proposes a method of identification.

Key words: collapsible soils, density index, consistency index, clay fraction.

$\mathrm{S}_{\mathrm{r}}=15,3 \%$, limite de liquidité $\mathrm{w}_{\mathrm{L}}=25,9 \%$, et indice de plasticité $I_{p}=9,9 \%$; les résultats des essais œdométriques sur sol saturé indiquent que les modules sont cinq fois inférieurs à ceux obtenus à l'état naturel, ce qui a conduit les ingénieurs à considérer que cette couche était effondrable.

La seconde couche, de 1,95 m d'épaisseur, est un limon argileux avec traces de sable. Les essais effectués montrent que ce sol présente un degré de saturation plus élevé, un poids volumique $\sec \gamma_{\mathrm{d}}=18 \mathrm{kN} / \mathrm{m}^{3}$ et une forte cohésion à l'etat sec. Ce sol a été considéré comme non susceptible à 'effondrement, vu la faible variation du module œdométrique; il perd cependant une grande partie de sa cohésion en cas de saturation.

La solution adoptée à Hassi-Messaoud a consisté à substituer la première couche par un matériau granulaire compacté et à prendre des mesures particulières pour éviter les infiltrations d'eau dans la seconde couche (LNHC Batna, 2000). 
Les sols effondrables sont des sols non saturés pouvant subir un réarrangement intergranulaire accompagné d'une diminution brusque et importante de leur volume après avoir été inondés. Les tassements excessifs qui en résultent ont motivé l'étude de ce type de sols. Certains chercheurs se sont penchés sur les méthodes d'identification et de traitement, comme Barden (1973), Jennings et Knight (1975) et Ayadat (1996, 1998). D'autres études, au contraire, ont été consacrées aux mécanismes d'effondrement (collapse en anglais) par Knight (1961), Barden et al. (1969), Ganéshan (1982), Ayadat et al. (1998), Cui et Magnan (2000).

Selon Dudley (1970), deux types d'effondrement peuvent se présenter. Dans le cas où la structure est cimentée, l'effondrement ne dépend plus de l'humidification, mais du niveau de contrainte appliquée. En revanche, si le sol est constitué de grains liés avec des matériaux fins (argiles ou limons) qui induisent des forces de liaisons importantes dues à la succion ou à la cimentation, l'humidification conduit à une annulation de la succion qui entraîne une diminution importante de la résistance et favorise ainsi l'effondrement, si la contrainte mécanique est suffisamment grande. Cette description du mécanisme d'affaissement a été confirmée par les travaux de Cui et Magnan (2000). En se basant sur le critère de Mohr-Coulomb généralisé, ces auteurs ont montré que l'effondrement est contrôlé par plusieurs facteurs, à savoir : la minéralogie des matériaux constitutifs, la fraction argileuse, la granulométrie, la teneur en eau naturelle, l'indice des vides, la dimension et la forme des pores, les éléments de cimentation ainsi que la nature et la concentration des ions dans l'eau interstitielle. Ceci confirme les conclusions de Dudley (1970). Morgenstern et De Matos (1975), Zur et Wiseman (1973) et Ganeshan (1982) ont démontré que les faibles teneurs en eau constituent l'une des principales causes de l'effondrement. Milovic (1969), Markin (1969), Booth (1975), Ting (1979) et Ganéshan (1982) ont mis en évidence l'influence importante de la densité sèche initiale, de l'indice des vides et du degré de saturation sur le comportement des sols effondrables. Markin (1969), Prusza et Choudry (1979) proposent une limite supérieure du degré de saturation initial $\left(S_{r}=60 \%\right.$ à $\left.65 \%\right)$ au-delà de laquelle l'affaissement n'apparaît plus. Les études menées par Mishu (1963), Booth (1975), (1977) et Ganéshan (1982) ont abouti à une limite du degré de saturation légèrement inférieure $\left(S_{r}=50 \%\right.$ à $\left.60 \%\right)$ et ont montré que le risque d'affaissement des sols ayant un degré de saturation supérieur à cette limite diminue considérablement. Dans ce cas, l'affaissement devient indépendant du chargement appliqué. Dans leurs études, Booth (1975), Cox (1978) et Lawton (1989) ont observé que, pour une densité sèche donnée, la surcharge pour laquelle l'effondrement est maximal est inversement proportionnelle à la teneur en eau naturelle du sol. Peu de travaux ont été développés jusqu'à présent quant à l'influence de l'indice de densité, de l'indice de consistance et du pourcentage des particules fines sur l'affaissement des sols. Le but de la présente recherche est la contribution à l'étude de l'influence de ces paramètres essentiels sur l'effondrement des sols.

\section{Matériaux et essais}

Les essais ont été effectués sur 11 sols reconstitués en laboratoire. Ces sols sont composés de sables et de particules fines en différentes proportions, pour lesquels l'application des différents critères d'effondrement, rapportés par Luttengeer et Saber (1988), montre une susceptibilité à l'effondrement. Les caractéristiques des sols reconstitués sont données dans le tableau I. Les courbes granulométriques sont représentées sur la figure 1. Le sable utilisé dans la reconstitution provient de la rivière de Liwa de la région de Biskra. Le sol fin (D $<80 \mu \mathrm{m})$ utilisé est extrait de Sidi-Maansar de la région de Timgad (Algérie). Ces sols ainsi reconstitués présentent de bonnes caractéristiques mécaniques lorsqu'ils sont à de faibles teneurs en eau $\left(\mathrm{w}_{0}=2 \%\right.$, $4 \%, 6 \%$ ). Cependant, à une contrainte constante de $200 \mathrm{kPa}$, une inondation engendre d'importantes variations de volume. Ce comportement est représenté par le diagramme de la figure 2. Jennings et Knight (1975) ont suggéré quelques valeurs du potentiel $\mathrm{C}_{\mathrm{p}}$ en fonction de l'importance de l'affaissement (voir tableau II).

La reconstitution est réalisée par compactage dans un moule œdométrique standard à une teneur en eau et un poids volumique sec fixés au préalable. Les essais sont effectués sur les sols à différentes teneurs en eau et indice de densité.

$$
I_{D}=\left(e_{\max }-e\right) /\left(e_{\max }-e_{\text {min }}\right)
$$

Les paramètres retenus sont:

- teneurs en eau : $2 \%, 4 \%$ et $6 \%$

- indices de densité: $10 \%, 30 \%$ et $50 \%$.

TABLEAU I Caractéristiques géotechniques des sols.

Geotechnical characteristics of soils.

\begin{tabular}{l|l} 
Matériaux & Caractéristiques \\
\hline Sable & $\begin{array}{l}\text { Équivalent de sable: } 70 \% \\
\text { Granulométrie comprise entre } 0,08 \text { et } 2 \mathrm{~mm}, \\
\text { Coefficient d'uniformité de } 2,5 \\
\text { Coefficient de courbure de } 0,56\end{array}$ \\
Fraction argileuse & $\begin{array}{l}\text { La fraction argileuse }(\%<2 \mu \mathrm{m}) \text { est de } 32 \% \\
\text { Limite de liquidité } \mathrm{w}_{\mathrm{L}}=41,50 \% \\
\text { Limite de plasticité } \mathrm{w}_{p}=28,9 \% \\
\text { Densité spécifique des grains } \mathrm{G}_{\mathrm{s}}=2,7\end{array}$
\end{tabular}

$\begin{array}{llllllllllll}\text { Sol } & \mathrm{S} 1 & \mathrm{~S} 2 & \mathrm{~S} 3 & \mathrm{~S} 4 & \mathrm{~S} 5 & \mathrm{~S} 6 & \mathrm{~S} 7 & \mathrm{~S} 8 & \mathrm{~S} 9 & \mathrm{~S} 10 & \mathrm{~S} 11 \\ \% \text { Sable } & 100 & 90 & 80 & 70 & 60 & 50 & 40 & 30 & 20 & 10 & 00 \\ \%<80 \mu \mathrm{m} & 00 & 10 & 20 & 30 & 40 & 50 & 60 & 70 & 80 & 90 & 100 \\ \mathrm{e}_{\max } & 0,765 & 0,773 & 0,817 & 0,828 & 0,846 & 0,913 & 0,955 & 1,007 & 1,035 & 1,184 & 1,472 \\ \mathrm{e}_{\min } & 0,458 & 0,452 & 0,436 & 0,408 & 0,403 & 0,396 & 0,383 & 0,367 & 0,350 & 0,333 & 0,327 \\ \mathrm{w}_{\mathrm{L}} \% & I & 13,7 & 15,1 & 16,1 & 19,3 & 21,4 & 26,8 & 34,0 & 34,3 & 40,6 & 41,5 \\ \mathrm{w}_{\mathrm{p}} \% & / & 8,4 & 10,9 & 11,4 & 11,9 & 13,6 & 15,2 & 22,0 & 22,6 & 23,7 & 28,9\end{array}$




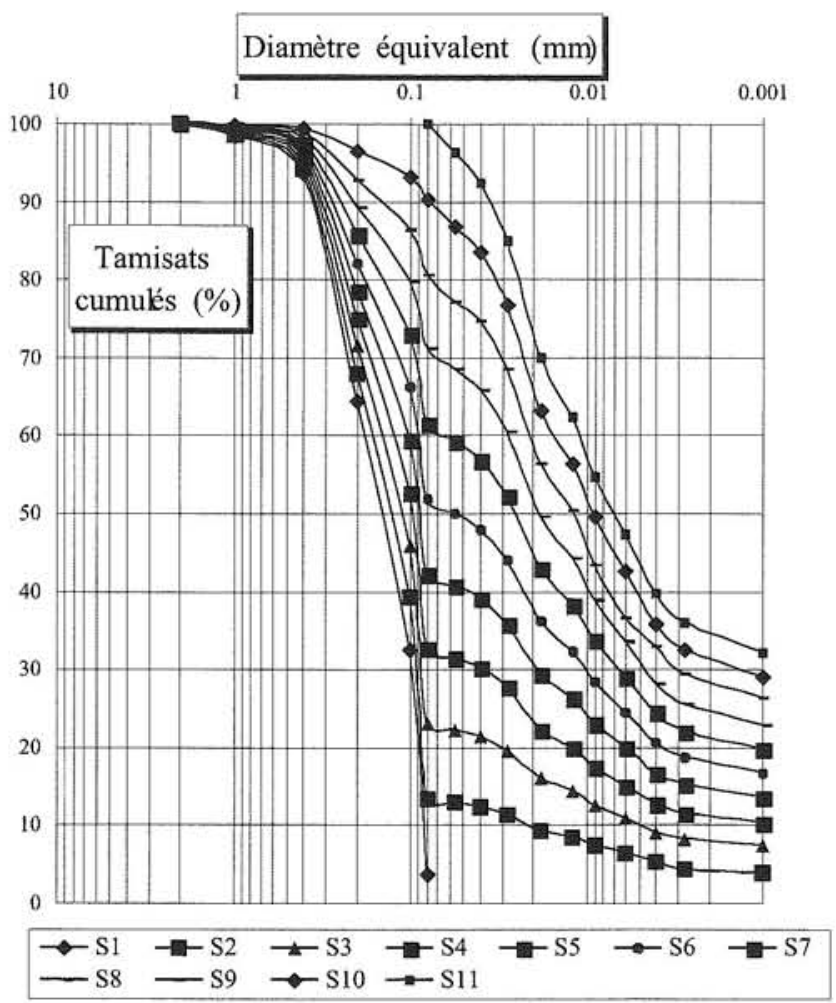

FIG. 1 Courbes granulométriques des matériaux. Grain size distribution curves.

\section{Résultats et analyses}

Une série d'essais a été réalisée dans le but de vérifier si ces sols possèdent les propriétés connues des sols effondrables naturels. Les résultats obtenus montrent clairement que les effondrements des différents sols s'inscrivent dans la classification de Knight et Jennings (1975). En effet, pour les sols S1 jusqu'au S11 les potentiels $\mathrm{C}_{\mathrm{p}}$ varient de $0,16 \%$ à $12,55 \%$ pour une teneur en eau $\mathrm{w}_{0}=2 \%$, de $0,13 \%$ à $5,73 \%$ pour une teneur en eau $\mathrm{w}_{0}=4 \%$ et de $0,045 \%$ à $3,63 \%$ pour une teneur en eau $\mathrm{w}_{0}=6 \%$. Ces résultats correspondent aux rubriques allant de ( pas de risque ) à ( troubles sévères ») (Tableau II). Par contre, quand la teneur en eau augmente, le potentiel d'affaissement décroît voire même s'annule au-dessus d'une certaine valeur. On a constaté que, pour une teneur en eau donnée, le potentiel d'effondrement décroît avec l'augmentation du poids volumique initial. Ces résultats concordent avec ceux de Lawton (1989), Barden et al. (1961) Holtz et Hilf (1961), Ayadat et al. (1998). De ce fait, on remarque que les sols préparés artificiellement possèdent un comportement analogue à ceux rencontrés in situ, ce qui justifie le programme d'essais adopté.

TABLEAU II Valeurs du potentiel d'effondrement selon Jennings et Knight (1975).

Values of collapse potential according to Jennings \& Knight (1975).

\begin{tabular}{l|l}
$\mathrm{C}_{\mathrm{p}}$ & Évaluation du risque \\
\hline $0 \%$ à $1 \%$ & Pas de risque \\
$1 \%$ à $5 \%$ & Troubles modérés \\
$5 \%$ à $10 \%$ & Troubles \\
$0 \%$ à $20 \%$ & Troubles sévères \\
$>20 \%$ & Troubles très sévères
\end{tabular}

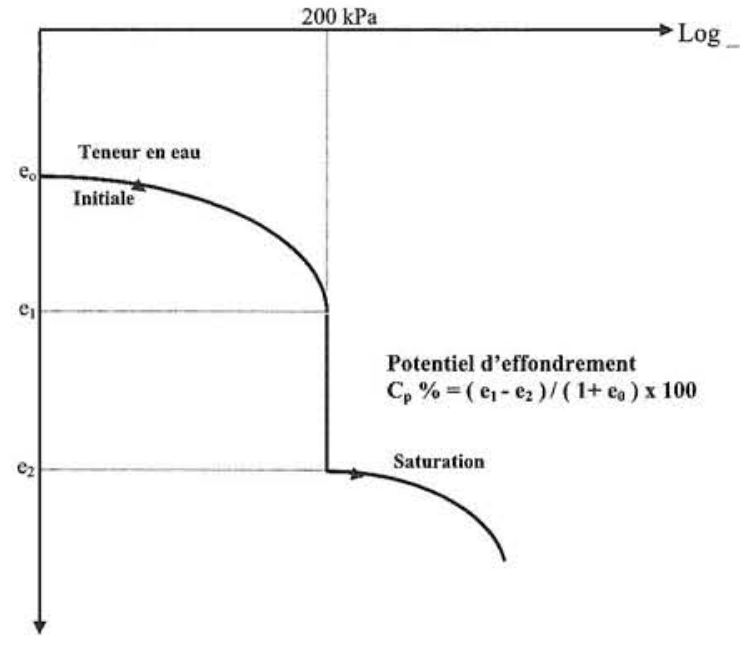

Indice des vides $\mathrm{e}$

FIG. 2 Courbe œdométrique typique d'un sol effondrable d'après Knight et Jennings (1975).

Typical oedometric curve of a collapsing soil (Knight and jennings 1975).

\subsection{Influence de I et I sur le coefficient de susceptibilité à l'effondrement $C_{p}$}

$L$ 'indice de liquidité $\mathrm{I}_{\mathrm{L}}$ est donné par la relation $\mathrm{I}_{\mathrm{L}}=$ $\mathrm{W}_{\mathrm{o}}-\mathrm{w}_{\mathrm{p}} / \mathrm{I}_{\mathrm{p}}$, où $\mathrm{w}_{\mathrm{o}}, \mathrm{W}_{\mathrm{p}}$ et $\mathrm{I}_{\mathrm{p}}$ sont respectivement la teneur en eau initiale, la limite de plasticité et l'indice de plasticité. Les résultats obtenus en utilisant cette relation pour les différents sols aux différentes teneurs en eau sont représentés par la figure 3 . On note qu'un sol est sujet au phénomène de l'effondrement si son indice de liquidité est négatif $\left(\mathrm{I}_{1}<0\right)$, ce qui confirme les résultats de Priklonskij (1952) et d'Ayadat et Ouali (1999).

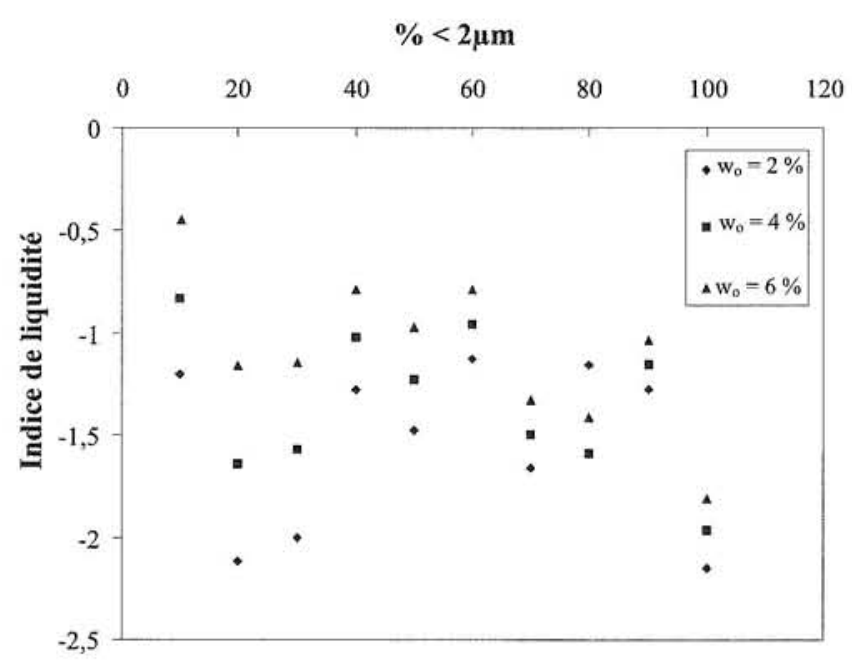

FIG. 3 Variation de l'indice de liquidité en fonction de la fraction argileuse $(\%<2 \mu \mathrm{m})$. Changes of the liquid index versus clay fraction. 


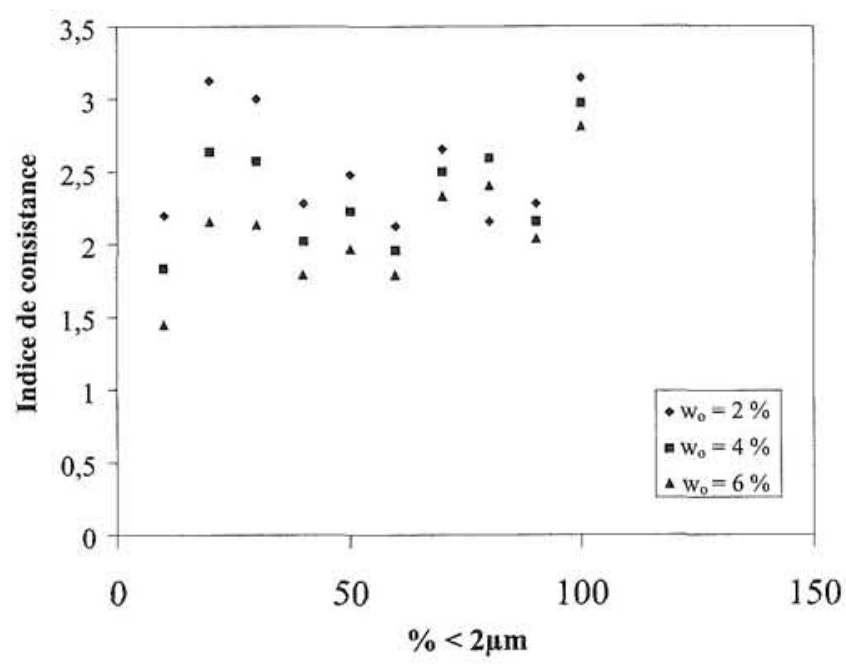

FIG. 4 Variation de l'indice de consistance en fonction de la fraction argileuse $(<2 \mu \mathrm{m})$. Change of the consistency index versus clay fraction.

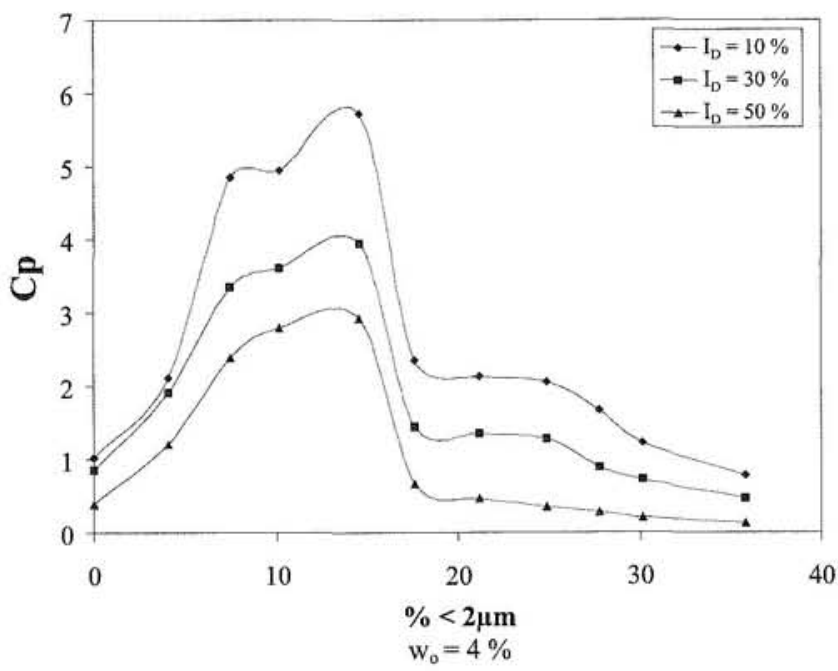

FIG. 5b Variation du potentiel d'effondrement $C_{p}$ en fonction de la fraction argileuse $(<2 \mu \mathrm{m})$. Change of the collapse potential $C_{p}$ versus clay fraction $(\%<2 \mu \mathrm{m})$.

En considérant l'indice de consistance $\left(\mathrm{I}_{\mathrm{c}}=1-\mathrm{I}_{\mathrm{L}}\right)$, on constate qu'un sol est susceptible de s'affaisser si son indice de consistance est supérieur à $1\left(\mathrm{I}_{\mathrm{C}}>1\right)$ comme le montre le graphique de la figure 4.

\subsection{Influence de l'indice de densité sur le coefficient de susceptibilité à l'effondrement $C_{p}$}

L'influence de l'indice de densité sur le potentiel d'affaissement des sols est présentée sur les figures $5 \mathrm{a}$, b, c. On constate que le coefficient $C_{p}$ décroît lorsque la compacité augmente, quels que soient le type de sol et la teneur en eau considérés. On remarque aussi que pour un indice de densité donné, le potentiel d'effon-

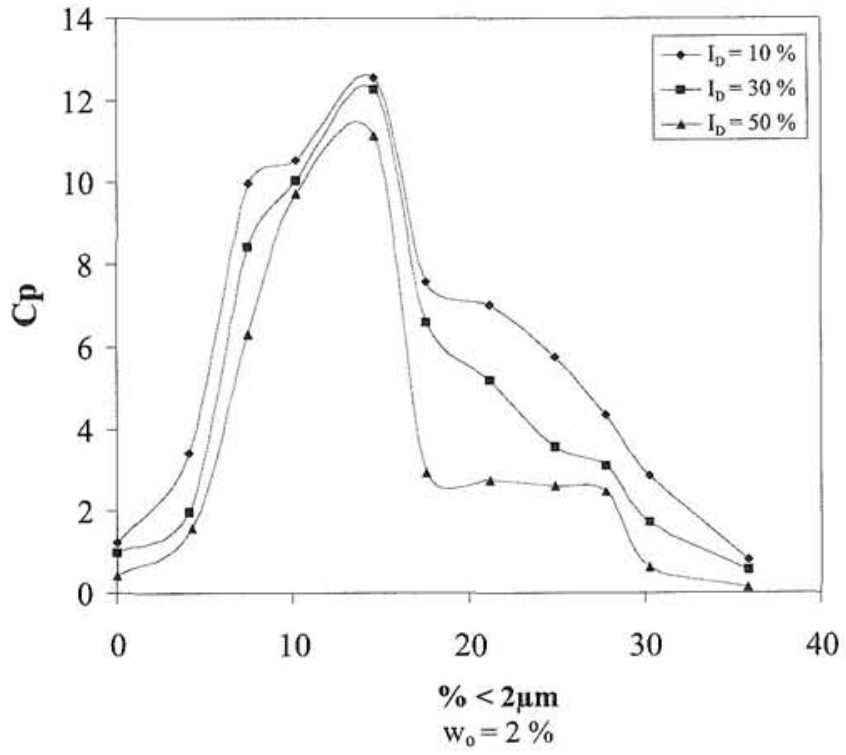

FIG. 5a Variation du potentiel d'effondrement $C_{p}$ en fonction de la fraction argileuse $(<2 \mu \mathrm{m})$.

Change of the collapse potential $\mathrm{C}_{\mathrm{p}}$ versus clay fraction $(\%<2 \mu \mathrm{m})$.

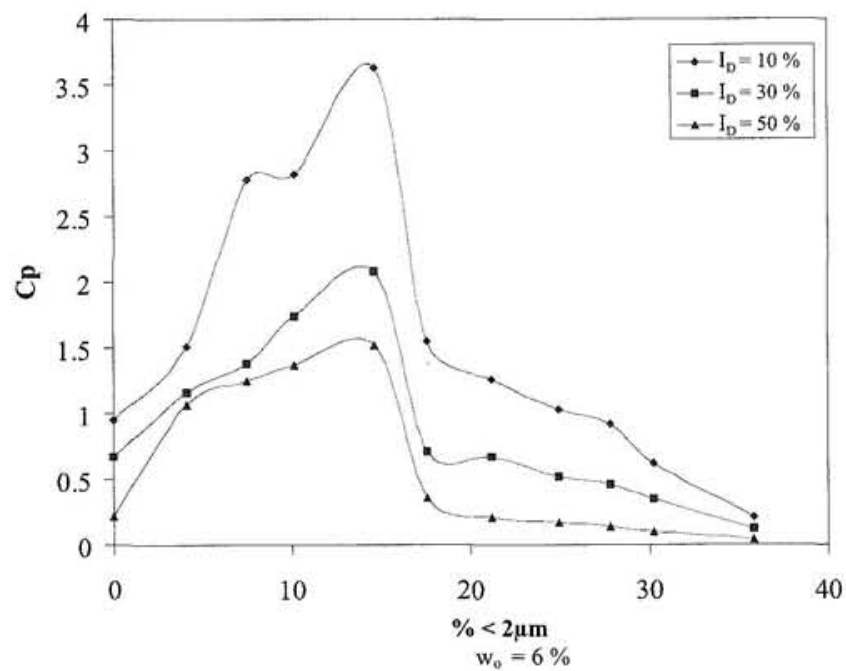

FIG. 5c Variation du potentiel d'effondrement $C_{\mathrm{p}}$ en fonction de la fraction argileuse $(<2 \mu \mathrm{m})$.

Change of the collapse potential $\mathrm{C}_{\mathrm{p}}$ versus clay fraction $(\%<2 \mu \mathrm{m})$.

drement diminue quand la teneur en eau augmente. Pour $\mathrm{I}_{\mathrm{D}}=50 \%$ et $\mathrm{w}_{\mathrm{o}}=6 \%$, voisine de la teneur en eau optimale de la majorité des sols étudiés, la figure $5 \mathrm{c}$ montre qu'à l'exception des quatre sols (S2, S3, S4 et S5 ), tous les sols testés ont un coefficient $C_{p}<1$ et sont donc non effondrables. De ce fait, on a jugé utile de tester ces derniers sols avec un indice de densité plus important $\left(I_{D}=70 \%\right)$. Les résultats obtenus sont présentés sur les figures $6, \mathrm{a}, \mathrm{b}, \mathrm{c}, \mathrm{d}$. On peut constater sur ces figures que, pour une teneur en eau $\mathrm{w}_{\mathrm{o}}=6 \%$, le potentiel d'effondrement est négligeable lorsque l'indice de densité $I_{D}$ est supérieur à $65 \%$. On note aussi que l'augmentation du potentiel d'affaissement est d'autant plus importante que le sol est lâche et avec une faible teneur en eau initiale. 


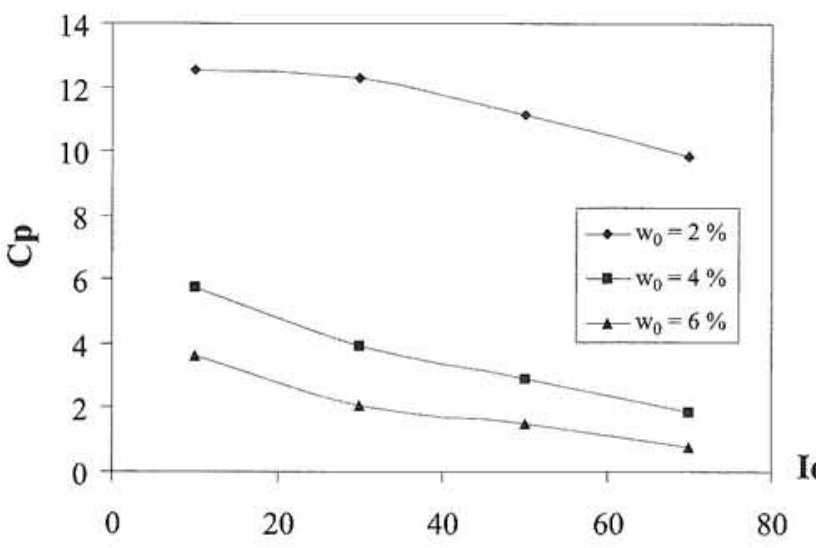

FIG. 6a Variation du potentiel d'effondrement $C_{p}$ en fonction de l'indice de densité $\mathrm{I}_{\mathrm{D}}$ pour le sol S5.

Collapse potential variation $C_{p}$ versus density index $I_{D}$, soil S5.

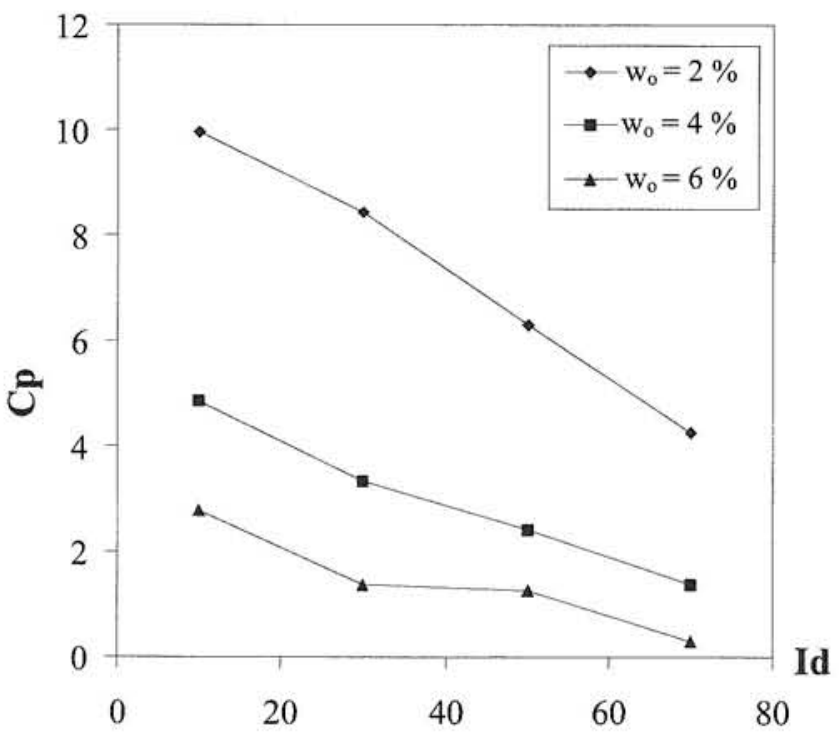

FIG. 6c Variation du potentiel d'effondrement $C_{p}$ en fonction de l'indice de densité $I_{D}$ pour le sol S3.

Collapse potential variation $C_{p}$ versus density index $\mathrm{I}_{0}$, soil S3.

\section{3}

\section{Influence des particules fines sur le coefficient de susceptibilité à l'effondrement $C_{p}$}

Les figures $5 \mathrm{a}, \mathrm{b}, \mathrm{c}$ illustrent l'influence des particules fines $(\%<2 \mu \mathrm{m})$ sur $\mathrm{C}_{\mathrm{p}}$. On constate que l'affaissement des sols dépend de la teneur en argile présente dans leur structure, ce qui confirme l'observation faite par Lawton et al. (1992). A partir de ces graphiques, il est clair que le potentiel de l'effondrement est négligeable quand le taux d'argile dans le squelette solide est supérieur à $30 \%$. Au-dessous de $5 \%$, il peut y avoir un effondrement qui demeure faible, tandis que le maximum d'effondrement est atteint au voisinage de $15 \%$. Ce résultat s'inscrit dans l'intervalle établi par Lawton et al. (1992) qui ont indiqué que le maximum du potentiel d'effondrement est obtenu lorsque la fraction argileuse est comprise entre $10 \%$ et $40 \%$. La clas-

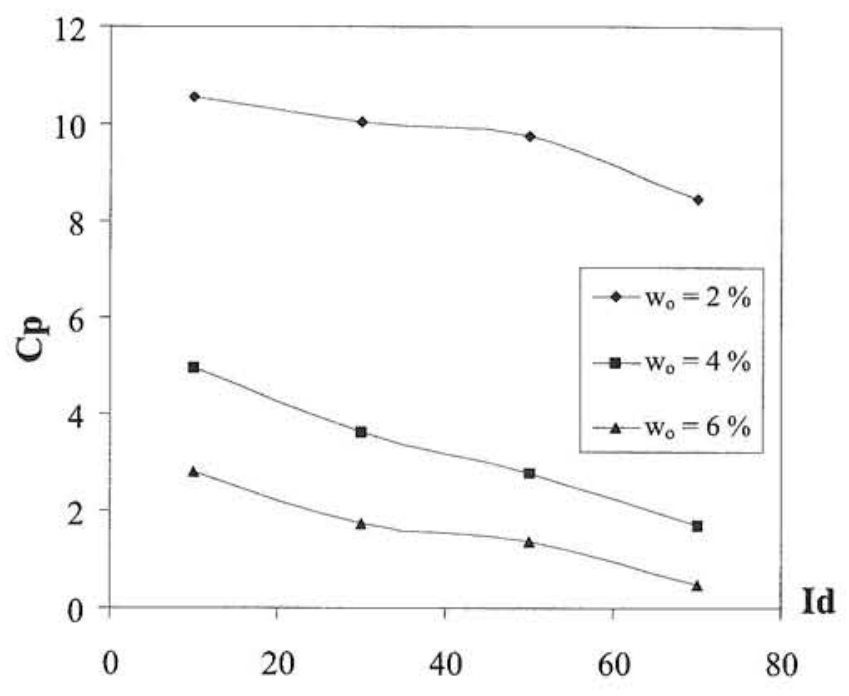

FIG. 66 Variation du potentiel d'effondrement $C$ en fonction de l'indice de densité $I_{D}$ pour le sol S4.

Collapse potential variation $\mathrm{C}_{\mathrm{p}}$ versus density index $\mathrm{I}_{\mathrm{D}}$, soil $\mathrm{S} 4$.

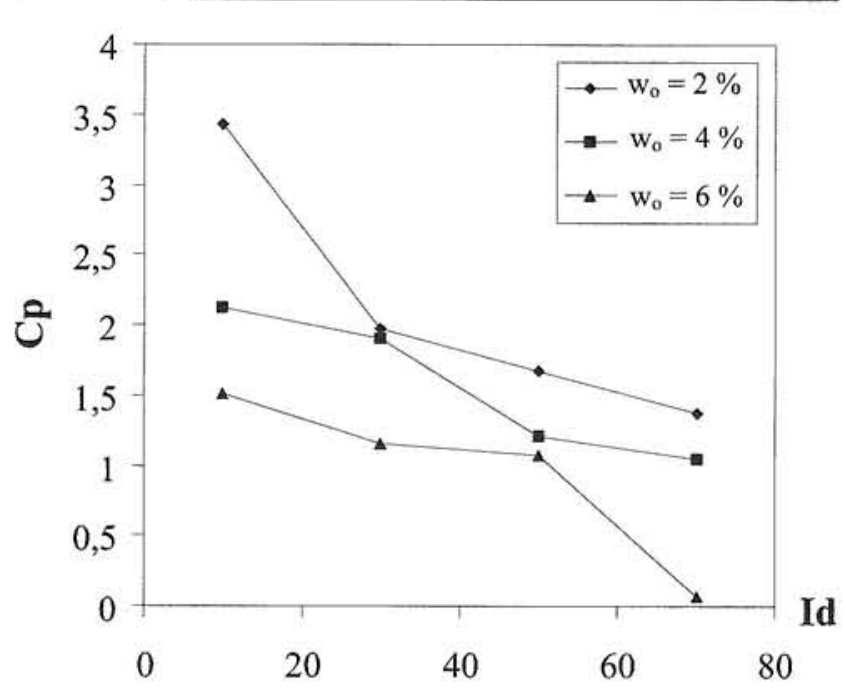

FG. $6 d$ Variation du potentiel d'effondrement $C$ en fonction de l'indice de densité Id pour le sol S2.

Collapse potential variation $\mathrm{C}_{\mathrm{p}}$ versus density index $I_{D}$, soil S2.

sification proposée ici sur le tableau III permet d'identifier les sols qui sont sujets au phénomène d'effondrement.

\section{Conclusion}

TABLEAUIII Classification proposée des sols effondrables.

Proposed classification for collapsible soils.

Fraction argileuse $(\%<2 \mu \mathrm{m})$, Susceptibilité limite de liquidité $\mathrm{w}_{\mathrm{L}}(\%)$

$5 \%<(\%<2 \mu \mathrm{m})<15 \%$

L'effondrement est très probable $\mathrm{w}_{\mathrm{L}}<20 \%$

$15 \%<(\%<2 \mu \mathrm{m})<30 \%$

$15 \%<\mathrm{w}_{\mathrm{L}}<30 \%$

L'effondrement est probable

$(\%<2 \mu \mathrm{m})>30 \%$

$\mathrm{w}_{\mathrm{L}}>35 \%$

Pas d'effondrement 
Les conclusions principales qu'on peut tirer de cette étude se résument comme suit:

- un sol est susceptible à l'effondrement si, au moins, l'un des critères suivants est vérifié : $I_{L}<0$ ou $I>1$; - on estime qu'un sol n'est pas effondrable si son indice de densité $\mathrm{I}_{\mathrm{D}}$ est supérieur à $65 \%$ avec une teneur en eau initiale proche de la teneur en eau optimale;
- le potentiel d'effondrement d'un sol dépend principalement de sa fraction argileuse $(\%<2 \mu \mathrm{m})$;

- il est possible de prévoir la susceptibilité d'effondrement d'un sol au laboratoire en se basant sur la granulométrie, d'une part, et les limites d'Atterberg, d'autre part, conformément aux résultats indiqués au paragraphe 3.3.

\section{Bibliographie}

Ayadat T., Belouahri B. - Influence du coefficient d'uniformité sur l'amplitude et le taux de l'affaissement des sols. Revue française de géotechnique, $\mathrm{n}^{\circ} 76$, $3^{\mathrm{e}}$ trimestre 1996, p. 25-34.

Ayadat T., Bensalem A., Benkerri A. - Traitement d'un sol affaissable par la chaux. Revue française de géotechnique, $\mathrm{n}^{\circ} 77$, 4 trimestre 1996 , p. $69-72$

Ayadat T., Belouahri B., Ait ammar R. - La migration des particules fines comme approche d'explication du mécanisme de l'effondrement des sols. Revue française de géotechnique, $\mathrm{n}^{\circ} 83,2^{\mathrm{e}}$ trimestre 1998, p. 1-9.

Ayadat T., Dahli M., Maiz A.K. - Traitement d'un sol effondrable par un liant hydrocarboné (bitume). Revue française de géotechnique, $\mathrm{n}^{\circ} 83,2^{\mathrm{e}}$ trimestre 1998 , p. 1-7.

Ayadat T., Ouali S. - Identification des sols affaissables basée sur les limites d'Atterberg. Revue française de géotechnique, $n^{\circ} 86,1^{\text {er }}$ trimestre 1999 , p. 53-56.

Barden L., Madedor A.O., Sides G.R. Volume change characteristics of unsaturated clay. Journal of SMFE, ASCE, vol. 95, SM1, 1969, p. 33-49.

Barden L., Mc Gown A., Collins K. - The collapse mechanism in partly saturated soil. Engineering Geology, 1973, p. 49-60.

Booth A.R. - The factors influencing collapse settlement in compacted soils. Proc. 6th Reg. Conf. for Africa on Soil Mech. and Found. Eng., Durban, South Africa, 1975, p. 57-63.

Booth A.R. - Collapse settlement in compacted soils. CSIR research report 321, NITRR Bulletin 13, Pretoria, South Africa, 1977.

Cox D.W. - Volume change of compacted clay fill. ICE Conf. on Clay fills, 1978, p. 79-86.

CTC Biskra - Intervention du directeur du contrôle technique de construction (CTC) de Biskra, $2^{\mathrm{e}}$ coll. maghrébin en génie civil, 10 et 11 décembre 2002, Biskra, Algérie.

Cui Y.-J., Magnan J.-P. - Affaissements locaux dus à l'infiltration d'eau en géomécanique environnementale. Chapitre $\mathrm{n}^{\circ} 6$ ( Risques naturels et patrimoine ». Ed. Hermès, 2000, p. 139-164.

Dudley J.H. - Review of collapsing soils. J. Soil Mech. and Found. Div., ASCE, 96, n SM3, 1970, p. 925-947.

Ganeshan V. - Strength and collapse characteristics of compacted residual soils. Thesis (M.E), Asian Institute of Technology, Bangkok, Thailand, 1982

Holtz W.G., Hilf J.W. - Settlement of soil foundation due to saturation. Proc. 5th Int. Conf. on Soil Mech. and Found. Eng., vol. 3, 1961, p. 673-679.

Jennings J.E., Knight K. - A guide to construction on or with materials exhibiting additional settlement due to collapse of grain-structure. Proc. 6th. Reg. Conf. on Soil Mech. and Found. Eng. for Africa, 1975, p. 99-105.

Knight K. - Collapsing behavior of residual soils. Proc. 7th Southeast Asian Geotech. Conf., Hong Kong, 1982, p. 915-930.

Lawton E.C., Fragaszy R.J., James H.H. Collapse of compacted clayey sand. J. of Geotech. Eng., ASCE, vol. 155, n 9 , 1989, p. 1252-1267.

Lawton E.C., Fragaszy R.J., Hetherington M.D. - Review of wetting-induced collapse in compacted soil. $J$, of Geotech. Eng., ASCE 118, n 9, 1992, p. 1376-1394. LNHC - Projet extension des gazoducs à
Hassi Messaoud - Rapport de reconnaissance de sol. Laboratoire national de l'habitat et de la construction de Batna, Algérie, 2000.

Lutenegger A.J., Saber R.T, - Determination of collapse potential of soils. Geotech. Testing J. 11, n 3 , 1988, p. 173178

Markin B.P. - Discussion on standard criteria of sag in loess soils. Soil Mechanics and Foundations Engineering, ${ }^{\circ} 2$ 2, 1969, p. 137.

Milovic - Engineering properties of loess and other collapsible structures. Proc. 7th Int. Conf.on Soil Mech. and Found. Eng., Moscow, 1969.

Mishu L.P. - Collapse in one-dimensional compression of compacted clay on wetting. MSc Thesis, Purdue University, at West Lafayette, 1963

Morgenstern N., De Matos M.M. Stability of slopes in residual soils. Proc, 5th. Pan American Conf, on Soil Mech. and Found. Eng., Buenos Aires. Argentina, 3,1975 , p. $367-$ 383.

Priklonskij V.A. - Gruntovedenie Vtoriaia Chast. Soil Science II Gasgedizdat, Moscow, 1952, p. 371.

Prusza A., Choudry T. - Collapsibility of residual soils. Proc. 13th Congress on Large Dams, New Delhi, India, Q. 49, R. 9, 1979, p. 117-130.

Ting W.A. - Consolidation of a partially saturated residual soil. Proc. 6th Asian Reg. Conf. on Soil Mech. and Found. Eng., Singapore, vol. 1, 1979, p. 95-98.

Zur A., Wieseman C. - Study of collapse phenomena of an undisturbed loess. Proc. 8th Int. Conf. on Soil Mech. and Found. Eng. Moscow, USSR, vol. 2 , part 2, 1973, p. 265-269. 